\title{
Normes de genre, violences sexuelles et vulnérabilité au VIH/sida en Nouvelle-Calédonie
}

\author{
Christine SALOMON et Christine HAMELIN ${ }^{1}$
}

\begin{abstract}
Résumé
Cet article s'intéresse à l'intersection des violences et du risque d'infection par le VIH chez les femmes kanakes de Nouvelle-Calédonie, dans un contexte où les taux de violences sexuelles sont élevés et s'enracinent à la fois dans des normes de genre encore très inégalitaires et dans une situation socio-économique défavorable empêchant l'autonomie des femmes de cette communauté. En raison d'un taux féminin d'infection à $\mathrm{VIH}$ encore relativement faible, la transmission directe par des rapports sexuels contraints ne peut être mise en évidence, mais la prise de risque est attestée par de mauvais indicateurs de santé sexuelle corrélés aux violences sexuelles subies précocement et par l'utilisation insuffisante de protection tant vis-à-vis du risque de grossesse non désirée que des maladies sexuellement transmissibles.
\end{abstract}

Mots clés : Kanaks, genre, violences sexuelles, risque, protection.

En Nouvelle-Calédonie, pour une population estimée à environ 230000 habitants comprenant $44 \%$ de Kanaks, $34 \%$ d'Européens (Calédoniens de souche européenne et métropolitains), $12 \%$ de Polynésiens (Calédoniens de souche polynésienne ou venant pour l'essentiel de Wallis et Futuna), $5 \%$ d'Asiatiques et $5 \%$ de personnes hors de ces groupes (" autres "), 295 cas de $\mathrm{VIH} /$ sida cumulés (dont 76 femmes) ont été déclarés, parmi lesquels 108 sida, dont 30 femmes, entre 1986 et fin $2006^{2}$. Les femmes représentent actuellement environ un tiers des personnes traitées et des nouveaux cas enregistrés. La distribution communautaire, bien que renseignée sur les fiches de déclaration, n'a jamais été communiquée par les autorités sanitaires, à la différence de celle des autres maladies transmissibles (dont celle des maladies sexuellement transmissibles, publiée jusque dans le milieu des années 1990). Une catégorie statistique nouvelle a été créée pour rendre compte de l'épidémie à VIH. Elle distingue les personnes qui ont voyagé de celles « qui n'ont jamais quitté le pays " (à savoir celles nés en Nouvelle-Calédonie et celles économiquement défavorisées, au premier chef desquels les Kanaks), dont la part dans les nouveaux cas n'a cessé de s'accroître.

Unité Inserm 687, Saint-Maurice et Université Paris XI, IFR 69, Villejuif. Source : DASS/CPS.
Une thèse de médecine montre cependant la composition communautaire de la file active (sujets soignés pour le $\mathrm{VIH} /$ sida et régulièrement suivis) fin 2001: $68 \%$ d'Européens, $15 \%$ de Kanaks, $10 \%$ de Polynésiens, $5 \%$ de personnes répondant " autre " à la question sur la communauté et $2 \%$ d'Asiatiques. La sur-représentation importante des Européens apparait liée aux modalités de transmission du VIH aux débuts de l'épidémie : les hommes ayant des relations avec d'autres hommes, dans leur grande majorité des Européens et des Polynésiens, totalisaient $40 \%$ des cas cumulés à cette date et les injecteurs de drogue, tous des Européens métropolitains, $6 \%$ des cas cumulés. En revanche, chez les Kanaks, la transmission avait été hétérosexuelle dans $75 \%$ des cas [1].

L'analyse des données statistiques officielles arrêtées fin 2006 (DASS/CPS) indique que, depuis 2000 , on compte autant de nouveaux cas féminins que masculins chez les moins de 30 ans. L'on ne sait si ce sont des femmes kanakes, mais celles-ci apparaissent particulièrement vulnérables du fait de la combinaison de facteurs qui tiennent à l'histoire coloniale, aux inégalités sociales, aux normes et aux violences de genre au sein de leur communauté, parfois tous englobés dans le concept holiste de violence structurelle. Les Kanakes sont $84 \%$ à vivre en milieu rural, alors que $83 \%$ des Européennes et $95 \%$ des Polynésiennes vivent dans le Grand Nouméa, la seule zone véritablement urbanisée du pays ${ }^{3}$. Bien qu'il existe, essentiellement en ville, un certain degré de brassage culturel et sexuel entre communautés, une nette superposition persiste entre clivages sociaux et communautaires : les Européennes, notamment métropolitaines, continuent de bénéficier d'une meilleure situation en termes d'éducation, d'emploi et de revenus que les Polynésiennes et surtout que les Kanakes.

Nos recherches ethnographiques en milieu kanak qui se sont intéressées au genre [2-6], aux représentations du sida et à la gestion du risque chez les femmes [7,8], ainsi qu'aux particularités des jeunes urbaines $[9,10]$ ont toutes mis en lumière le poids des violences dans la vie des femmes. Mais pour mesurer, à l'échelle du pays, l'ampleur des violences et comparer de ce point de vue les différentes communautés, nous avons entrepris, dans le prolongement de l'enquête nationale sur les violences envers les femmes en France (Enveff), une enquête en

Source : enquête Santé, conditions de vie et de sécurité des femmes calédoniennes (2002-2003). 
population générale féminine "Santé, conditions de vie et de sécurité des femmes calédoniennes ${ }^{4}$. Bien que l'échelle d'observation, la nature des données à traiter et du raisonnement explicatif diffèrent, la combinaison de l'ethnographie et des statistiques [11] s'est révélée particulièrement utile pour comprendre les violences de genre, dont les déterminants relèvent à la fois de l'histoire individuelle et familiale, de normes culturelles et de facteurs socio-économiques et pour montrer qu'en NouvelleCalédonie la santé sexuelle se décline très différemment selon la communauté d'appartenance des femmes.

La prévalence de l'infection est insuffisante ${ }^{5}$ pour mettre en évidence un lien statistique direct entre contrainte sexuelle et transmission du VIH, alors que c'est là un trait important de l'épidémie dans des pays de forte prévalence tels que l'Ouganda et l'Afrique du Sud ${ }^{6}$. Cependant, en indiquant comment les normes inégalitaires et les violences hypothèquent la capacité des femmes kanakes à négocier les actes sexuels et à se protéger, l'exemple calédonien souligne la relation entre violences de genre et VIH et plaide pour les faire reconnaitre comme des co-épidémies se développant dans les mêmes contextes culturels, sociaux et politiques [15-17].

\section{Lutte anti-coloniale, théories autochtones et sida}

Lorsque le sida fit son apparition en Nouvelle-Calédonie, l'information à ce sujet dans les sociétés kanakes fut réappropriée grâce aux catégories servant à penser la maladie et la contagion, qui ne procèdent pas d'un système figé mais plutôt d'un processus de réflexion sur les aléas de l'existence dans lequel la recherche des origines du mal constitue un paradigme. Deux théories, apparemment contradictoires, firent rapidement leur apparition pour expliquer les origines et la transmission du sida.

La première considérait la maladie avec sa part d'inconnu comme imputable à l'Autre (il était en effet de notoriété publique que le premier cas identifié en 1986 était un militaire français ${ }^{7}$ ) et s'en distanciait en la classant dans la catégorie des « maladies des Blancs ", aux côtés des autres épidémies dues au contact avec l'Occident. En Kanaky (Nouvelle-Calédonie), comme en Irian Jaya (Papouasie occidentale) [18], le contexte de soulèvement nationaliste et d'occupation militaire massive du pays était favorable aux explications persécutives quant à l'introduction de la maladie. Des indépendantistes décrivaient le sida en termes de " facteur de génocide biologique » et interprétaient le refus des autorités sanitaires de communiquer la répartition communau-

\footnotetext{
Cette étude sur les violences à l'encontre des femmes a été menée auprès d'un échantillon représentatif de 1012 femmes de 18 à 54 ans, interrogées en face à face au moyen d'un questionnaire standardisé.

5 Chez les femmes accouchées récentes, elle était de 0,66 pour mille en 2000 [12]

De plus, dans ces pays, des travaux soulignent la surexposition aux violences conjugales des femmes vivant avec le VIH $[13,14]$

7 Dans le Pacifique, la Nouvelle-Calédonie, la Polynésie française et Guam, trois pays avec une forte présence militaire et une population importante non autochtone, sont les premiers à avoir rapporté des cas d'infection par le VIH.
}

taire des cas comme une mesure politique discriminatoire visant à empêcher les Kanaks de se protéger. De plus, l'accent mis dans le seul quotidien local, Les Nouvelles Calédoniennes et dans les émissions télévisées sur les groupes à risque, prostituées et homosexuels, renforçait la construction d'une maladie due à la contagion et à la perversité occidentales. En effet à cette époque, la plupart des Kanaks percevaient l'homosexualité comme étrangère à leur société et la prostitution elle-même comme une institution importé $e^{8}$. Dans les différentes langues kanakes, il n'existe pas de terme pour désigner l'homosexualité ni la prostitution, et l'on parle des prostituées en utilisant l'expression "épouses de la route ", qui stigmatise les femmes adultères et, par extension, toutes celles qui utilisent leur sexualité en dehors des règles de la circulation matrimoniale.

Une autre construction du sida l'assimilait non pas à une " maladie des Blancs ", mais à une " maladie indigène " déjà connue, de la catégorie des maladies sexuellement transmissibles elles-mêmes subdivisées en maladies sexuellement transmissibles sèches et humides. Le sida était vu comme une maladie " sèche ", c'est-à-dire sans écoulement, mais avec des signes généraux, tels la fièvre et la toux. Cela ouvrait sur une explication dans laquelle les femmes étaient à l'origine du mal, dans la mesure où elles auraient contaminé les hommes en transgressant les interdits sexuels pendant les règles, la grossesse et le post-partum. Dans plusieurs langues du Nord, les maladies sexuellement transmissibles sont désignées au moyen du terme servant à nommer la case des femmes (différente autrefois de celle des hommes) et un élu kanake d'une cinquantaine d'années chargé de la santé dans cette Province devait d'ailleurs énoncer (en français) lors d'une émission télévisée en 1993 que le sida était une "maladie des femmes". Cette construction était véhiculée principalement par les personnes d'un certain âge, les hommes surtout, les femmes étant quant à elles peu nombreuses à adhérer à cette logique d'imputation du risque.

Mais dans ces années où la revendication identitaire kanake était à son apogée, le poids du contexte politique sur les constructions de la maladie était tel que les deux logiques, catégorisation et imputation, se combinèrent rapidement pour faire du sida, surtout en milieu rural, une maladie des femmes qui fréquentaient les Blancs et, de ce fait, se trouvaient en rupture à la fois avec les normes de genre et avec la lutte d'indépendance. Le sida aurait ainsi été transmis par des jeunes femmes kanakes venues vivre en ville parce qu'elles avaient choisi le célibat ou étaient avec un partenaire non kanake ou, pire encore, étaient engagées dans des liaisons avec des militaires français. Sur ces femmes, stigmatisées, non seulement comme de mauvaise vie mais aussi comme traîtres à la cause, circulaient des rumeurs les accusant de propager la maladie. Un scénario récurrent dans le Nord mettait en

\footnotetext{
$8 \quad$ Parmi les femmes prostituées, des Indonésiennes ou des Tahitiennes, figurent néanmoins aussi quelques Kanakes, plutôt occasionnelles que régulières. La prostitution régulière est en majorité masculine et composée pour l'essentiel de travestis polynésiens.
} 
scène un jeune homme qui allait à Nouméa, y rencontrait dans une boîte de nuit une fille kanake qui " traînait » avec des militaires ou des marins, avait avec elle une aventure sexuelle et, de retour chez lui, contaminait d'autres partenaires féminines [8]. Dans cette reconstruction, la responsabilité originelle de la contamination est encore imputée à une femme, mais néanmoins externalisée, dans la mesure où sa résidence urbaine et ses pratiques de mixité sexuelle la rendent étrangère au groupe. La peur de la stigmatisation et du rejet due à ces logiques éclaire sans doute encore presque dix ans plus tard le secret gardé par les personnes séropositives, en particulier dans les zones rurales où l'interconnaissance est extrêmement forte. En 2003, dans l'enquête en population générale féminine "Santé, conditions de vie et de sécurité des femmes calédoniennes ", les femmes kanakes vivant en milieu rural étaient très peu nombreuses $(3,5 \%)$ à connaître quelqu'un de séropositif comparées aux femmes kanakes vivant dans le Grand Nouméa (10\%).

Bien que ces idées sur la maladie n'aient pas totalement disparu, elles ne sont plus aujourd'hui véhiculées par l'élite politique kanake. La contestation grandissante des normes de genre par les femmes et, au-delà, par de nombreux jeunes (filles et garçons) a eu pour effet de saper l'assimilation du sida à une " maladie des femmes". De même, la théorie de l'introduction intentionnelle du sida pour décimer le peuple kanake a disparu de la presse nationaliste et a régressé dans l'opinion après que, Iors d'une émission télévisée pour la journée mondiale du sida, en 1995, un pasteur kanake, leader reconnu de l'Église évangélique protestante qui elle-même s'était prononcée en faveur de l'indépendance, ait révélé que son fils aîné était récemment mort du sida. Sa déclaration contribua à déconstruire le sida comme maladie des Blancs, d'autant plus puissamment que jusque-là en Nouvelle-Calédonie aucun Européen n'avait témoigné à visage découvert de sa séropositivité (c'est d'ailleurs encore le cas aujourd'hui). Elle intervenait de surcroît dans un contexte politique qui s'apaisait avec la gestion institutionnelle par les Indépendantistes des Provinces Nord et des Îles Loyauté et dans un moment où s'opérait, avec l'introduction des antirétroviraux, un glissement dans la perception de la maladie elle-même.

\section{Contrainte sociale et santé sexuelle}

Dans les sociétés kanakes, traditionnellement, l'organisation sociale repose sur un principe hiérarchique qui régitégalement les normes de genre : les gens du commun sont subordonnés à ceux de qualité, les cadets aux aînés, les jeunes aux vieux et encore davantage les femmes aux hommes. Mais alors que les lignages de moindre importance peuvent accéder à un rang supérieur au terme de stratégies politiques complexes, que les cadets peuvent espérer voir disparaître les aînés et que les jeunes vont devenir vieux un jour, les femmes, au nom du biologique et de leur impureté, sont exclues des sphères du pouvoir [2-5]. Comme Mary
Douglas l'a montré, l'impureté supposée des femmes joue un rôle dans le maintien de la domination masculine [19]. La notion même de droits des femmes, introduite par l'administration française en Nouvelle-Calédonie en 1989, a rencontré une hostilité telle que dans le Nord lui fut substituée l'appellation Mission de la femme qui, par l'utilisation du singulier, essentialise les femmes et évite l'idée dérangeante de droits. Plus récemment, la parité politique a soulevé un débat lui aussi révélateur : tandis que les associations de femmes soutenaient son application, bon nombre de leaders kanakes, qu'ils soient indépendantistes ou pro-français, s'y opposèrent. En dépit d'avancées notables, particulièrement chez les femmes les plus jeunes et chez celles qui vivent en ville, le patriarcat reste davantage légitimé que chez les Européennes. Dans l'enquête en population générale féminine "Santé, conditions de vie et de sécurité des femmes calédoniennes ", les Kanakes sont $38 \%$ à considérer que c'est à l'homme de commander dans la famille ( $14 \%$ chez les Européennes), $19 \%$ à justifier les coups si la femme est soupçonnée d'infidélité (1 \% chez les Européennes) et $15 \%$ si elle ne remplit pas ses tâches ménagères (aucune Européenne). C'est chez les rurales, qui sont les plus pauvres et les moins éduquées, que la légitimation de la domination masculine est la plus enracinée.

Les entretiens non directifs réalisés dans le cadre d'enquêtes ethnographiques ${ }^{9}$ avec des femmes kanakes, dont les plus âgées sont nées dans les années 1920, font ressortir, dans les générations précédentes, l'usage systématique de la contrainte sociale et de la violence physique pour obliger les filles à accepter des unions non voulues. Malgré une régression incontestable des mariages arrangés, $20 \%$ parmi les répondantes kanakes mariées de l'enquête "Santé, conditions de vie et de sécurité des femmes calédoniennes " déclarent que ce sont les parents qui ont choisi leur conjoint. L'éducation prépare d'ailleurs les jeunes à choisir leur partenaire dans un groupe social pré-déterminé, si bien qu'aujourd'hui le choix du conjoint reste largement dicté par les règles d'alliance : dans $84 \%$ des mariages, l'union est approuvée et sanctionnée par les deux groupes de parenté intéressés, celui de la femme et celui de son conjoint. On n'observe pas de différence significative selon l'âge et le niveau d'éducation des femmes, ce qui révèle la force des normes matrimoniales kanakes.

Jusque récemment, une femme ne pouvait pas sortir d'une union malheureuse et le divorce était exceptionnel. En cas de conflit, la femme généralement ne pouvait que repartir pour un répit dans sa propre famille, stratégie qui perdure puisque une femme kanake sur six (17\%) vivant en couple s'est déjà séparée de son conjoint plus de trois mois, un taux deux fois supérieur à celui des Européennes (8\%).

En dépit d'une tendance à l'individualisation des conduites chez les jeunes, les femmes dont le style de vie est perçu comme

Ces enquêtes menées depuis 1990 par C Salomon ont successivement abordé les questions de la vie reproductive et sexuelle, les représentations de la maladie et du sida, les normes de genre et la perception de la justice. 
trop libre demeurent exposées à la critique et à un certain ostracisme social en milieu rural. Enfreindre les règles est condamné et le refus des relations sexuelles peut être assimilé à une infraction si l'homme est considéré comme en droit de les imposer, qu'il soit le mari réel ou potentiel de la femme. Comme de nombreuses autres langues mélanésiennes [20,21], les langues kanakes rassemblent sous une même appellation, qui peut se traduire par "relations hors normes ", le viol, l'adultère et les relations consenties avec un partenaire que les règles d'alliance désapprouvent.

\section{Des violences sexuelles fréquentes}

Le viol lors du premier rapport sexuel apparaît relativement banalisé, d'autant qu'il n'existe pas de norme de virginité prémaritale. Une femme kanake sur huit (12\% versus 2,4 \% chez les Européennes) déclare un premier rapport sexuel forcé sans qu'il y ait de différence significative selon l'âge. Par ailleurs, les viols et les tentatives de viol de jeunes adultes commis par des hommes de l'entourage (autres que le conjoint) au cours des douze derniers mois ne sont pas des exceptions, surtout chez les femmes rurales et chez celles sans revenus personnels : $12 \%$ des Kanakes âgées de 18 à 24 ans déclarent ce type de violences sexuelles, ce qui est bien plus élevé que chez les Européennes de Nouvelle-Calédonie ( $5 \%$ ), elles-mêmes cinq fois plus exposées que les femmes en France [22].

II faut également noter la persistance des viols collectifs [4-6], désignés en français par l'euphémisme " la chaîne ". Les victimes sont le plus souvent des jeunes femmes quelque peu marginalisées par leur style de vie ou qui consomment de l'alcool jusqu'à être ivres lors des fêtes. Leur profil donne à ces viols un aspect de punition des filles qui ne se conforment pas aux normes de genre. L'âge, de 15 à 25 ans, le nombre des auteurs, qui peut atteindre dix, et la banalisation des faits leur confère également une dimension d'initiation sexuelle des garçons.

La socialisation des garçons à l'exercice de la violence et des filles à la soumission explique aussi que les épouses ne sont pas supposées se refuser sexuellement à leur conjoint. La proportion de femmes kanakes qui déclarent des viols conjugaux est élevée : $14 \%$ de celles ayant un partenaire régulier $(1,4 \%$ chez les Européennes). Le taux apparait significativement plus élevé chez les femmes mariées qui n'ont pas choisi librement leur conjoint ( $31 \%$ versus $12 \%$ ) et chez celles qui, à un moment donné, s'en sont séparées temporairement ( $24 \%$ versus $12 \%$ ). En revanche, les femmes kanakes vivant avec un partenaire non kanake, le plus souvent des urbaines, sont tendanciellement moins exposées que les autres $(7 \% \text { versus } 15 \%)^{10}$.

\footnotetext{
10 Ce résultat concorde avec les propos de nombreuses jeunes femmes kanakes sur l'avantage de se trouver un * Blanc * $[9,10]$.
}

\section{Maternité et contraception}

La vie en couple n'est en principe reconnue qu'après la naissance d'un enfant. Autrefois, l'enfantement déterminait l'accès au mariage et l'obtention pour la femme de droits dans le lignage de son époux. Jusqu'à maintenant, le mariage n'est généralement célébré qu'après la naissance d'un ou de plusieurs enfants, parmi lesquels on espère un garçon pour perpétuer le lignage du père. Néanmoins, à l'heure actuelle, surtout chez les jeunes, se fait jour une préférence pour le concubinage plutôt que le mariage. En effet, si le concubinage place la femme dans une situation de moindre reconnaissance, il permet de quitter le conjoint, ce qui devient très difficile une fois les échanges de mariage accomplis. Le déclin relatif de l'institution du mariage laisse toutefois intacte l'obligation d'enfanter, à laquelle veillent les hommes, maris ou concubins : $7 \%$ des femmes kanakes ( $10 \%$ aux Îles) déclarent que leur conjoint les a empêchées d'utiliser une contraception, alors que les Européennes sont moins de $2 \%$ dans ce cas. Les données sur l'âge à la première naissance mettent également l'accent sur les différences entre communautés : plus d'un tiers des femmes kanakes qui ont des enfants $(38 \%)$ ont eu leur premier-né avant l'âge de 20 ans, une proportion deux fois plus importante que les Européennes (18\%). Et bien que les grossesses précoces régressent avec le meilleur niveau d'éducation atteint depuis quelques années par les filles kanakes, $25 \%$ des Kanakes de 18 à 24 ans qui ont déjà donné naissance à un enfant se sont trouvées enceinte avant 18 ans.

Nos résultats mettent également en évidence un taux frappant de grossesses non désirées, y compris chez les plus jeunes. Parmi les femmes âgées de 18 à 44 ans et qui ont déjà eu au moins une grossesse, les Kanakes sont près de trois fois plus nombreuses que les Européennes à déclarer que leur dernière grossesse n'était pas désirée ( $22 \%$ contre $8 \%$ ). Chez les jeunes de 18 à 24 ans, les Kanakes représentent $79 \%$ des déclarations de dernière grossesse non désirée, ce qui atteste une difficulté à se protéger et à négocier les actes sexuels. De plus, quel que soit l'âge, presque une femme kanake sur cinq qui a voulu interrompre sa grossesse n'a pu le faire. L'apparente contradiction entre le taux de grossesses non désirées plus élevé et le taux d'interruption de grossesse plus faible que dans les autres communautés mérite qu'on s'y arrête. Pendant longtemps il n'y a pas eu de planning familial, la loi Veil n'est applicable que depuis 1995 en Nouvelle-Calédonie ${ }^{11}$ et le seul centre d'interruption volontaire de grossesse se trouve à Nouméa. Des entretiens ethnographiques menés auprès de jeunes femmes du Nord indiquent que le déclin des méthodes traditionnelles de limitation des naissances [2,3] n'a pas été compensé par l'accès aux contraceptifs modernes et à l'interruption volontaire de grossesse. Dans la mesure où consulter au dispensaire ne représente pas une garantie suffisante de confidentialité pour les jeunes femmes en milieu rural,

\footnotetext{
11 II faut signaler, entre autres raisons, l'opposition à l'avortement des églises locales, tant protestante que catholique.
} 
Figure 1

Taux de dépistage volontaire selon les caractéristiques sociodémographiques ( $n=939$ )

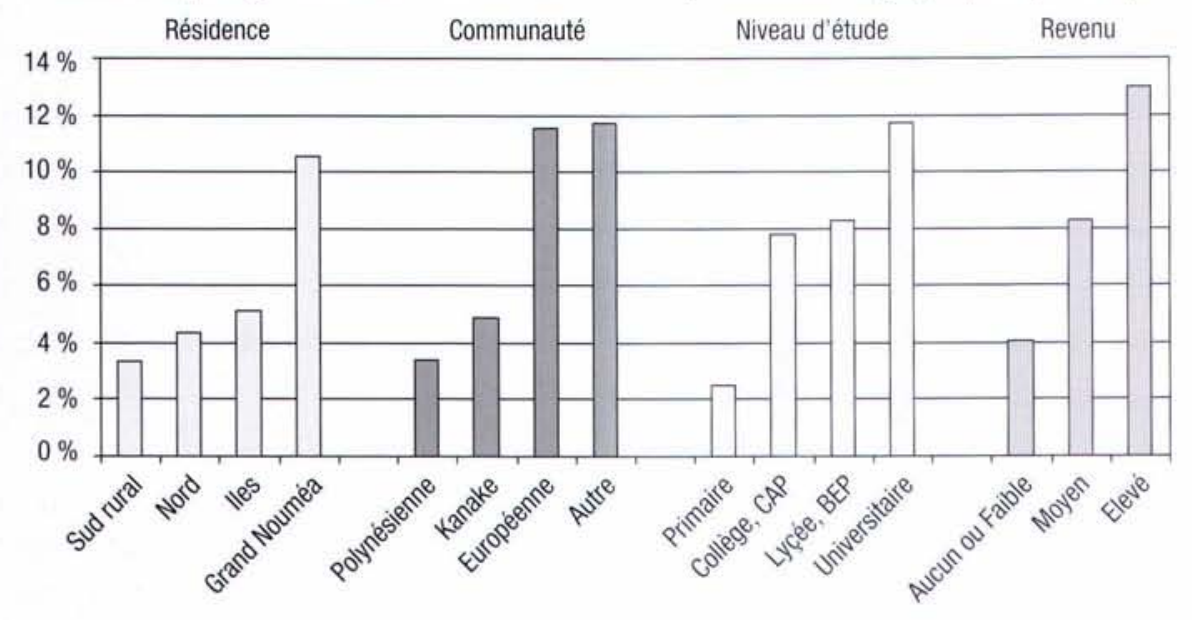

qu'elles n'ont pas les moyens de se rendre à Nouméa, elles n'ont souvent comme solution que de cacher leur grossesse le plus longtemps possible [23]. Les résultats de l'enquête par questionnaire confirment les difficultés des femmes à maîtriser leur vie reproductive et à se protéger. La contraception reste insuffisamment utilisée : $33 \%$ des femmes kanakes âgées de 18 à 44 ans n'utilisent ni pilules ni stérilet ni préservatif. En revanche, le taux de maladies sexuellement transmissibles est élevé : $6 \%$ des Kanakes en déclarent une au cours de l'année (contre 0,8\% des Européennes), un taux préoccupant dans la mesure où ces infections sont généralement sous-déclarées et certaines d'entre elles passent inaperçues.

\section{Dépistage et protection}

La moitié des femmes sexuellement actives $(51 \%)$ a pratiqué au moins un test, dans $55 \%$ des cas lors de la surveillance d'une grossesse, et dans seulement $15 \%$ des cas de sa propre initiative. II existe toutefois un écart considérable, en particulier dans les provinces rurales kanakes du Nord et des îles, entre les femmes qui déclarent un test et celles qui ont eu une grossesse depuis 1994 (date de l'offre du test) et auraient dû par conséquent se voir proposer un test : $53 \%$ des femmes qui auraient pu être testées durant la grossesse aux Îles et $45 \%$ de celles qui auraient pu l'être dans le Nord déclarent n'avoir jamais fait de dépistage. Cela ne signifie pas que les Kanakes sont dépistées de façon significativement moins fréquente que les autres, mais plutôt qu'elles n'identifient pas dans le prélèvement sanguin qui leur est fait la finalité de dépistage du VIH, probablement parce que le terme " sida " n'est pas utilisé par le praticien et que le sigle VIH n'est pas compris. Par conséquent, leur consentement n'apparaît pas réellement éclairé, ce qui rejoint d'autres observations sur le contenu « inadapté et mal compris » des entretiens pré-test [12]. Dans le Sud où se trouve Nouméa, l'écart entre grossesse et connaissance du dépistage (22\%) est deux fois plus fréquent chez les Kanakes (et les Polynésiennes) que chez les Européennes. Les tests volontaires, à l'initiative propre de la femme, apparaissent également bien moins fréquents chez les Kanakes (et les Polynésiennes) que chez les Européennes, le niveau d'éducation et de revenus des femmes étant avec la communauté un déterminant du test volontaire (figure 1).

Les normes qui attribuent aux hommes la prise de décisions pèsent également sur la capacité des femmes à imposer le préservatif. L'étude ethnographique sur la gestion du risque avait souligné qu'un certain nombre d'entre elles, surtout des rurales, considéraient que le préservatif était " une affaire d'hommes " et que celles qui en comprenaient la nécessité en avaient « en réserve " mais ne pouvaient le proposer de peur de passer pour des filles faciles. Á l'exception de quelques jeunes femmes titulaires du bac, qui localement est encore considéré comme un diplôme élevé, exiger la protection paraissait hors de question [8].

Les résultats de l'enquête par questionnaire confirment, à plus large échelle, ces données. La moitié des femmes sexuellement actives en Nouvelle-Calédonie (49 \%) n'a jamais utilisé le préservatif. Chez les Kanakes comme dans les autres communautés, les plus jeunes (18-24 ans), celles avec le niveau scolaire le plus élevé et, dans une moindre mesure, celles avec le revenu personnel le plus conséquent sont plus nombreuses à utiliser le préservatif. Néanmoins, de façon générale, les Kanakes (et encore davantage les Polynésiennes ${ }^{12}$ ) apparaissent nettement moins utilisatrices de préservatifs que les Européennes (figure 2).

Les femmes kanakes avec un nouveau partenaire dans l'année sont deux fois moins nombreuses à s'être protégées que les

Les Polynésiennes sont en majorité catholiques pratiquantes. Globalement, l'usage du préservatif est corrélé à la religion et, chez les catholiques, il diminue avec la régularité de la pratique religieuse. 
Figure 2

Taux d'utilisatrices de préservatif dans la vie, selon les caractéristiques sociodémographiques $(\mathrm{n}=939$ )

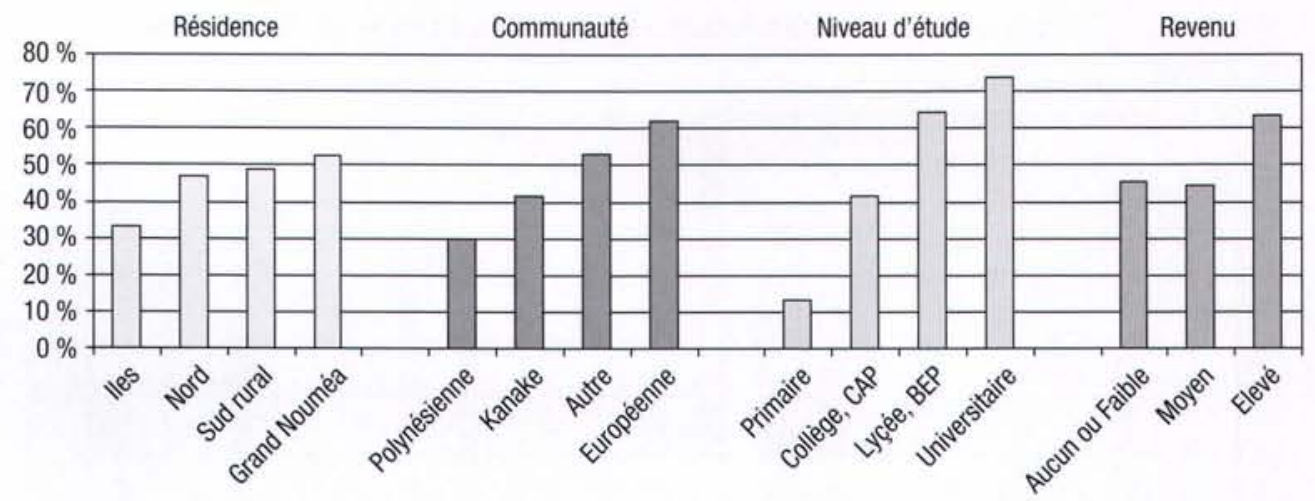

Européennes ( $36 \%$ contre $72 \%$ ). La différence communautaire est toutefois moins prononcée chez les jeunes: les moins de 25 ans utilisent deux fois plus souvent le préservatif que leurs aînées, ce qui suggère une certaine homogénéisation des comportements de prévention chez les jeunes.

Mais santé sexuelle et prise de risque à l'âge adulte apparaissent également conditionnées par les violences sexuelles subies dans l'enfance et l'adolescence. En empêchant les femmes de négocier ultérieurement les actes sexuels, les abus précoces constituent un facteur déterminant de vulnérabilisation au VIH.

\section{Violences sexuelle précoces et risque du VIH}

Avant l'âge de 15 ans, une femme sur huit (12\%) a vécu au moins une fois un attouchement, une tentative de viol ou un viol, les auteurs étant le plus souvent des hommes de la famille. Tandis que les autres expériences négatives dans l'enfance (les coups, le fait de vivre avec des parents alcooliques ou qui se battent) sont plus fréquentes chez les Kanakes (et dans une mesure moindre chez les Polynésiennes) que chez les Européennes, le niveau des violences sexuelles précoces est le même dans toutes les communautés. Cependant, alors qu'il diminue chez les jeunes Européennes en comparaison de leurs aînées, il augmente chez les jeunes Kanakes par rapport aux plus âgées. Parmi les Kanakes de 18 à 24 ans, $19 \%$ ont subi des violences sexuelles avant 15 ans, ce qui permet de faire l'hypothèse que les bouleversements actuels des structures familiales sont propices aux négligences et aux abus.

Le suivi des sessions d'assises en Nouvelle-Calédonie avait attiré notre attention sur le nombre de filles adoptées parmi les victimes kanakes ${ }^{13}$. Les agresseurs étaient, dans ces cas, soit le

\footnotetext{
13 Les transferts d'enfants constituent chez les Kanaks de Nouvelle-Calédonie, comme dans de nombreuses sociétés d'Océanie, une pratique très courante. Ils peuvent être plus ou moins formalisés et répondent à diverses situations dont le célibat de la mère. L'enquête en population générale féminine montre que $28,5 \%$ des femmes kanakes ont été dans leur enfance adoptées ou * données *.
}

parent adoptif soit un autre homme ayant autorité, généralement le nouveau compagnon de la mère. Les plus exposées paraissent être les filles nées de mères célibataires, généralement adoptées par un oncle utérin ou un grand-père maternel, ainsi que celles qui, sans être véritablement adoptées, sont simplement données à garder pour des raisons pratiques, telle la proximité de l'école par exemple, à un parent de leur mère ou vivent avec leur mère et le nouveau partenaire de celle-ci. Autrefois, ces enfants ne suivaient pas leur mère si elle se mariait et partait habiter chez son nouveau conjoint, mais restaient vivre chez leurs grandsparents ou leurs oncles maternels dont ils portaient le nom [23]. Or, de plus en plus souvent, les changements dans la parentalité et dans les conditions de vie induites par l'économie de marché conduisent des mères seules à garder avec elles leurs enfants, même si ceux-ci ont été adoptés quand elles emménagent avec un compagnon. Les données quantitatives confirment les observations ethnographiques, en attestant la surexposition significative au viol avant l'âge de 15 ans des Kanakes adoptées (7\% versus $2 \%$ chez les non adoptées).

L'ensemble des recherches sur les violences faites aux femmes démontre que les abus sexuels dans l'enfance constituent un facteur d'exposition aux violences ultérieures, en particulier celles du conjoint ou d'un autre homme de la famille, que la victimisation peut continuer sans interruption de l'enfance et l'adolescence jusqu'à l'âge adulte [24] et jouer un rôle négatif dans la gestion de la sexualité et du risque du VIH. Bien que les mécanismes par lesquels les violences subies dans l'enfance retentissent sur la vie sexuelle adulte soient complexes et encore mal connus, nos résultats, ainsi que ceux d'études menées en Inde et au Bangladesh [25, 26], indiquent que les abus sexuels précoces tendent à provoquer un dégoût ou un désintérêt de l'activité sexuelle. Quelle que soit leur communauté en Nouvelle-Calédonie, les victimes de violences sexuelles dans l'enfance refusent bien plus fréquemment des rapports à leur conjoint que celles qui n'ont pas vécu ces expériences ( $16 \%$ versus $6 \%$ ). Ce refus peut, en retour, susciter des actes contraints et violents, surtout dans le 
cadre du mariage. Les femmes qui déclarent refuser souvent des rapports sexuels à leur conjoint subissent près de cinq fois plus de viol marital que les autres ( $27 \%$ contre $6 \%$ ).

Les victimes d'abus sexuels dans l'enfance déclarent également davantage de rapports sexuels non voulus $(7 \%$ contre $4 \%$ ) et plus de maladies sexuellement transmissibles au cours de l'année $(7,5 \%$ contre $2,5 \%)$. Elles déclarent aussi significativement plus de grossesses non désirées ( $22 \%$ contre $9 \%$ ) et d'avortements ( $14 \%$ contre $6 \%)$. Tous ces éléments, qui concordent avec les résultats d'une série d'études menées aux États-Unis et en Afrique sub-saharienne [27-29], constituent des indicateurs de non-protection en faveur de l'hypothèse d'un continuum de risques, les abus sexuels précoces favorisant l'exposition aux violences sexuelles ultérieures et rendant incapable de se protéger.

\section{Conclusion}

Pour comprendre la faible marge de négociation sexuelle des femmes kanakes dont nous avons montré qu'elle était liée à des normes culturelles anciennes, il faudrait encore examiner la brutalité de la domination coloniale qui, au-delà des relations entre communautés, s'est étendue aux relations de genre. Le déséquilibre profond du sex ratio de la population immigrant en NouvelleCalédonie jusqu'à la seconde moitié du $\mathrm{xx}^{\mathrm{e}}$ siècle $^{14} \mathrm{a}$ contribué à la contrainte exercée par les colons et les libérés du bagne sur les femmes kanakes. L'impact de cette permissivité sexuelle à l'encontre des filles et des femmes autochtones, caractéristique des sociétés coloniales, sur les représentations et les comportements aujourd'hui mériterait plus d'attention. De même, il faudrait s'intéresser davantage aux Calédoniennes de souche européenne qui, dans le colonat libre, souvent isolées dans un habitat rural très dispersé, ont vécu de leur côté également une domination patriarcale forte. Le niveau de violences sexuelles subies par les Calédoniennes de souche européenne, plus élevé qu'en France, paraît influencé par cette culture coloniale connue en Australie sous l'appellation frontier culture.

Actuellement, en dépit du tournant post-colonial et du rééquilibrage en matière politique, sociale, juridique et sanitaire en faveur des Kanakes, la pauvreté continue de limiter l'accès des femmes à l'éducation et aux ressources et permet à des normes de genre, dont certaines seraient en passe de devenir obsolètes, de se maintenir ainsi que l'indiquent l'ampleur des violences et les indicateurs de santé reproductive et sexuelle particulièrement mauvais chez les femmes rurales. L'identification des facteurs à différents niveaux, culturel, social, familial ou personnel qui les fragilisent montre que c'est en synergie qu'il faut en NouvelleCalédonie s'attaquer aux violences à l'encontre des femmes et promouvoir la prévention du VIH et qu'il serait important d'inscrire

\footnotetext{
14 En 1921, les femmes représentaient seulement $25 \%$ de la population européenne et dans les zones rurales $20 \%$ de la population totale [30].
}

les inégalités de genre, et en particulier les violences sexuelles, au cœur même des interventions et des politiques de lutte contre le sida.

\section{Remerciements}

L'étude Santé, conditions de vie et de sécurité des femmes calédoniennes a reçu le soutien de l'Anrs, d'Ensemble contre le sida, de la Mission interministérielle de lutte contre les toxicomanies, du Gouvernement et des trois provinces de Nouvelle-Calédonie. Nous remercions France Lert, Marcel Goldberg. Alice Guéguen, Rémi Sitta, Pâquerette Goldberg, Diane Cyr et Jean-Pierre Nakache de l'unité Inserm 687, pour leur collaboration à l'étude par questionnaire et nos collègues du groupe de recherche 2835 du CNRS (Nouvelle-Calédonie : Enjeux sociaux contemporains) pour leur soutien.

\section{Références bibliographiques}

1. Isch JF. L'épidémie de VIH/Sida en Nouvelle-Calédonie. Le point au 31 décembre 2001. Thèse de médecine non publiée, 2003, Strasbourg.

2. Salomon C. La personne et le genre au Centre Nord de la Grande Terre (Nouvelle-Calédonie), Gradhiva 1998; (23) : 81-100.

3. Salomon C. Savoirs et pouvoirs thérapeutiques kanaks. Paris : PUF, 2000a.

4. Salomon C. Hommes et Femmes: harmonie d'ensemble ou antagonisme sourd? In En pays kanak, A Bensa, I Leblic, Eds. Mission du Patrimoine ethnologique, cahier 14. Paris: Ed. de la Maison des sciences de l'homme, 2000b, p. 311-38.

5. Salomon C. A'jië and Paicî Kanak Societies, a Women's Perspective : Obligatory Maternity and Reproductive Autonomy. In Birthing in The Pacific: Beyond Tradition and Modernity ? M Jolly, V Lukere, Eds. Honolulu : University of Hawaï Press, 2002, p. 99-124

6. Salomon C. Quand les filles ne se taisent plus : un aspect du changement postcolonial en Nouvelle-Calédonie. Terrain 2003a; 40:133-50.

7. Salomon-Nékiriai C. Le sida dans les sociétés kanakes : maladie des femmes et/ou maladie des Blancs? Journal des Anthropologues 1997 ; (68-69) : 129 42.

8. Bougerol C, Salomon C. Représentations du sida et gestion du risque chez les femmes kanakes de Nouvelle-Calédonie. Rapport CERMES-Anrs, 1998.

9. Hamelin C. Les gens de Nouméa : mutations et permanences en milieu urbain. In En pays kanak. Ethnologie, linguistique, archéologie, histoire de la NouvelleCalédonie, A Bensa, I Leblic, Eds. Paris : Mission du Patrimoine Ethnologique, Éditions de la Maison des Sciences de l'Homme, Ethnologie de la France, $n^{\circ} 14,2000$ a, p. 339-354.

10. Hamelin C. Histoire et enjeux contemporains de l'urbanisation kanake. Chroniques du Pays Kanak, Nouméa, Planète Mémo 2000b ; 4 : 2-7.

11. Weber $F$. « L'ethnographie armée par les statistiques ". Enquête 1995; 1 : 153-165.

12. Berlioz-Arthaud A, Baumann F. Seroprévalence du VIH chez les femmes enceintes de Nouvelle-Calédonie : bilan d'une année de surveillance. Bull Soc Pathol Exot 2002; 95(2) : 109-114.

13. Koenig M, Lutalo T, Zhao F et al. Coercive sex in rural Uganda : Prevalence and associated risk factors. Soc Science Med $2004 ; 58(4)$ : 787-798. 
14. Dunkle K, Jewkes R, Brown H et al. Prevalence and Patterns of Gender-based Violence and Revictimization among Women Attending Antenatal Clinics in Soweto, South Africa. Am J Epidem 2004 ; 160 (3) : 230-239.

15. Heise L, Moore K, Toubia N. Sexual coercion and reproductive health : a focus on research. New York : Population Council, 1995.

16. Petchesky R. Sexual rights : Inventing a concept, mapping an international practice. In Framing the sexual subject: The politics of gender, sexuality, and power, R Parker, RM Barbosa, P Aggleton, Eds. Berkeley and Los Angeles : University of California Press, 2000, p. 82-103.

17. Tabet $P$. La grande arnaque : L'expropriation de la sexualité des femmes Actuel Marx $2001 ; 30: 131-52$

18. Butt L. Lipstick Girls and Fallen Women : AIDS and Conspiratorial Thinking in Papua, Indonesia. Cultural Anthropology $2005 ; 20(3): 412-442$.

19. Douglas M. Purity and Danger : an analysis of the concepts of pollution and taboo. London : Routledge \& Kegan Paul, 1966.

20. Strathern M. Introduction. In Domestic Violence in Papua New Guinea, S Toft, Ed. Monograph $n^{\circ} 3$, Law reform Commission of Papua New Guinea, Port Moresby, 1985, p. 1-13.

21. Banks C. Contextualising sexual violence : rape and carnal knowledge in Papua New Guinea. In Reflections on Violence in Melanesia, S Dinnen, A Ley, Eds. Canberra : Hawkins Press and Asia Pacific Press, 2000, p. 83-104.

22. Jaspard $\mathrm{M}$, Brown $\mathrm{E}$ et al. Les violences envers les femmes en France. Une enquête nationale. Paris : La documentation française, 2003.
23. Salomon C. Maternité et transformations sociales. In Approches autour de Culture et Nature dans le Pacifique Sud, $\mathrm{H}$ Mokaddem, Ed. Nouméa: Expressions, 2003b, p. 61-83.

24. Hamelin C, Salomon C. Parenté et violences faites aux femmes en NouvelleCalédonie : un éclairage sur l'ethnicité différentiée des violences subies au sein de la famille. Espace, Populations, Sociétés $2004 ; 2$ : 307-23.

25. Khan ME, D'Costa S, Rahman M. Prevalence and nature of violence against women in Bangladesh. The 129th Annual Meeting of the American Public Health Association, Atlanta, GA, October 21-25, 2001.

26. Khan ME, Barge S, Sadhwani H. Reflections on Marriage and Sexuality; Experience of Newly Married men and Women in Gujarat, India. Vadodara, India, Centre for Operations Research and Training, 2004.

27. Maman S, Campbell J, Sweat MD, et al. The intersections of HIV and violence directions for future research and interventions. Soc Science Med $2000 ; 50$ $459-478$

28. Baleta A. Study reveals extent of rape in spread of HIV/AIDS. Lancet Infect Dis $2006 ; 6(12): 761$.

29. Editorial. Microfinance, intimate-partner violence, and HIV. Lancet 2006; $368: 1937$.

30. Merle I. Expériences coloniales : La Nouvelle-Calédonie, 1853-1920. Paris : Belin, 1995. 